Brit. Heart f., 1967, 29, 83.

\title{
Congenital Mitral Stenosis
}

\author{
S. P. SINGH ${ }^{\star}$, M. S. GOTSMAN, L. D. ABRAMS, R. ASTLEY, C. G. PARSONS, \\ AND K. D. ROBERTS
}

From the Heart Unit, Birmingham Children's Hospital

We have studied 8 children with congenital mitral stenosis and a left ventricle of approximately normal size. Of these, 4 are known to have had endocardial fibro-elastosis, 2 had a persistent ductus arteriosus, 1 had aortic stenosis, and 1 had an abnormal inferior vena cava. The presenting symptoms were recurrent respiratory infections, failure to thrive, orthopnœea, and dyspnoea. There was no history suggestive of rheumatic fever or disseminated lupus erythematosus.

\section{CASE Reports}

Case 1. As an infant this girl had repeated pulmonary infections and was noted to have an abnormal heart. When investigated in 1951 at the age of 4 years she was small (weight $30 \mathrm{lb}$. (13.6 kg.)). She had differential cyanosis with clubbing of her toes but pink, normal finger-tips. The heart was in sinus rhythm, slightly enlarged, and there was a diastolic thrill at the apex. In the mitral area there was an extremely loud first sound, and mid-diastolic and presystolic murmurs, strongly suggestive of mitral stenosis. The electrocardiogram (Fig. 1) and radiological appearances were consistent with the diagnosis. Venous angiocardiography showed that the right ventricle emptied normally into the pulmonary artery, from which contrast medium passed directly to the descending aorta, confirming reversed flow through a ductus arteriosus. The left atrium was greatly enlarged and extremely slow to empty. In 1952 the mitral valve was dilated by the digital method. The cusps tore rather than split; the valve felt leathery and appeared to have no commissures. The pulmonary arterial pressure, taken with a saline manometer at the time of operation, was $145 \mathrm{~cm}$. As valvotomy had little effect on this pressure it was decided not to close the ductus arteriosus. Biopsy of the left atrial appendix showed moderate fibro-elastosis of the endocardium.

Considerable symptomatic improvement followed and was maintained for several years. Then breathlessness gradually increased and differential cyanosis became

Received March 11, 1966.

* In receipt of a research grant from the Endowment Fund of the United Birmingham Hospitals. more obvious. Signs of mitral stenosis persisted and the pulmonary arterial pressure remained high (90/50 $\mathrm{mm}$. $\mathrm{Hg}$, in 1959). Eventually, at the age of 13, atrial fibrillation and cardiac failure developed and response to medical treatment was unsatisfactory. A second valvotomy using cardiopulmonary bypass was attempted in 1959. The re-stenosis was confirmed, but unfortunately she died during the procedure and permission for necropsy could not be obtained.

Case 2. A boy of 7 months was admitted in 1952 with a history of cough and failure to thrive. He was pale and wasted, weighing $10 \mathrm{lb} .8 \mathrm{oz}$. (4762 g.). The heart was enlarged. Presystolic and systolic murmurs were heard at the apex of the heart. Dyspnoea and signs of congestive heart failure were only temporarily relieved by digitalis and mersalyl. Radiographs showed cardiac enlargement with vascular engorgement of the lung fields, and the electrocardiogram (Fig. 1) showed changes suggestive of right ventricular hypertrophy. Venous angiocardiography supported the diagnosis of mitral stenosis, as the left atrium was large and slow to empty. The mitral valve was dilated surgically, but 36 hours later the child choked and died almost immediately. At necropsy (Dr. H. S. Baar) the diagnosis of mitral stenosis was confirmed. There was endocardial fibro-elastosis of the left atrium and upper part of the left ventricle, with widespread atelectatic areas in both lungs.

(Cases 1 and 2 were included in the report by Bower et al., 1953.)

Case 3. This boy was noticed to have a murmur at the age of 9 months; he had had recurrent bronchitis and had failed to thrive. When investigated in 1951 at the age of 3 years he was small (weight $25 \mathrm{lb}$. $(11.3 \mathrm{~kg}$.), height 32 in. $(81 \mathrm{~cm})$.$) , with a prominent sternum and$ recession of the lower ribs. He was dyspnœic, but not cyanosed. The heart was in sinus rhythm with a tapping apical impulse. The mitral first and pulmonary second sounds were loud, and a short early systolic murmur, a presystolic murmur, and a mid-diastolic murmur were heard at the apex. The electrocardiogram showed 83 


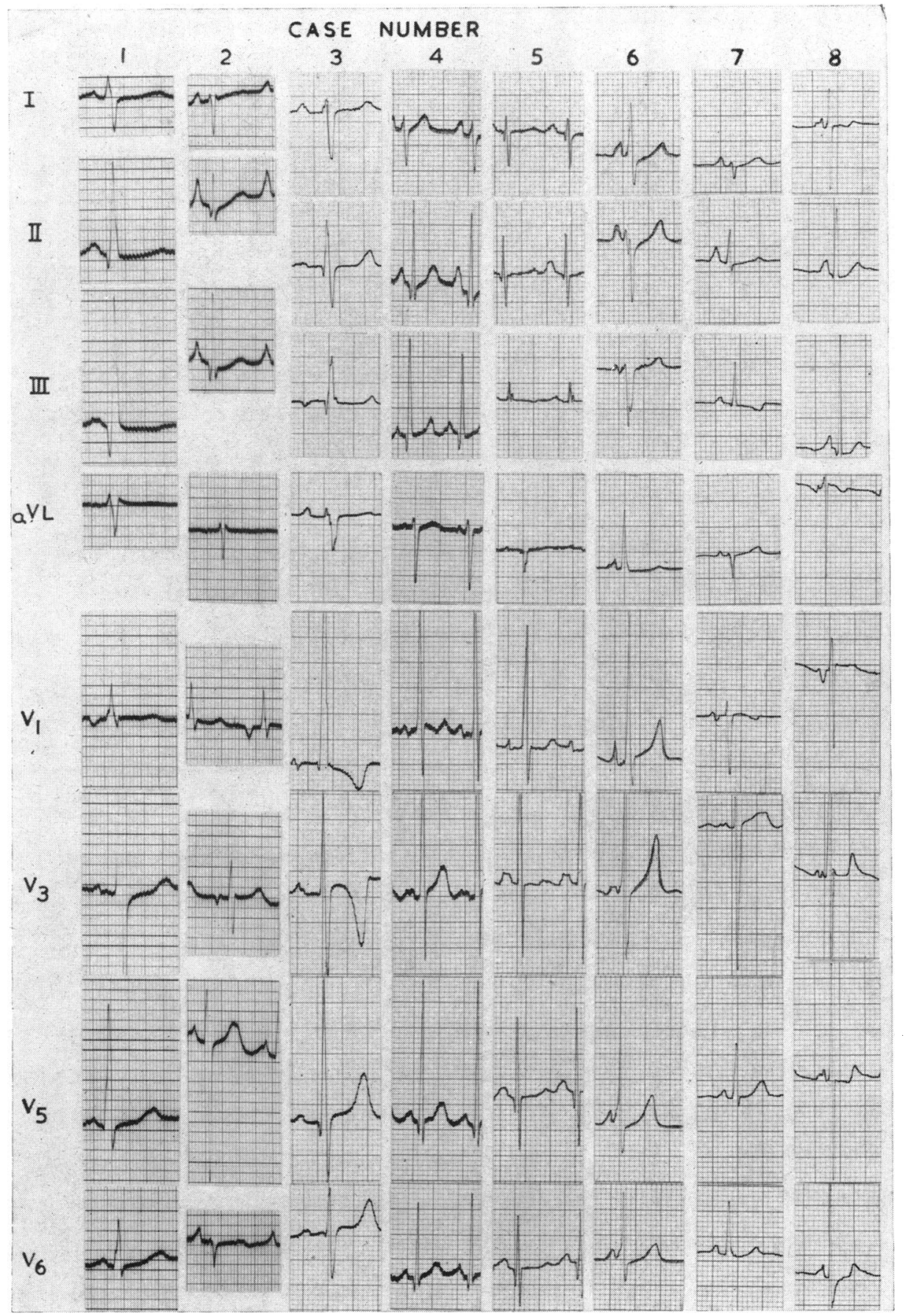

FIG. 1.-The electrocardiograms in 8 cases of congenital mitral stenosis.

hypertrophy (Fig. 1). Enlargement of the right ventricle and left atrium was confirmed by radiograph, the pulmonary artery segment was full and the lung vessels were congested. At the age of 5 an exploratory thora- cotomy was undertaken but the left atrium was small and the intended valvotomy was abandoned. The pulmonary arterial pressure was $40 / 15 \mathrm{~mm}$. Hg. Heart failure developed but was controlled with digitalis and diuretics. 
At the age of 10 catheterization was repeated. The pulmonary wedge pressure was $18 \mathrm{~mm}$. $\mathrm{Hg}$ and pulmonary arterial pressure $50 / 15 \mathrm{~mm}$. Hg. Selective right ventricular cine-angiocardiography showed a large, slowly emptying left atrium and a left ventricle of normal size.

In 1962 at the age of 16 years mitral valvotomy was performed under direct vision using cardiopulmonary bypass. This reduced the left atrial pressure from $40 / 2$ to $15 / 2 \mathrm{~mm}$. Hg. Three years after the operation he had no symptoms and the only abnormal sign was a faint apical systolic murmur.

Biopsy of the atrial appendix showed moderate myocardial hypertrophy but no endocardial fibro-elastosis.

Case 4. This boy had several attacks of bronchopneumonia in infancy and a murmur was heard at the age of 4 months. In 1963, when he was 4 years, he was investigated because of breathlessness on exertion, and orthopnœa. He was small (weight $34 \mathrm{lb} .(15 \cdot 4 \mathrm{~kg}$.), height 38 in. $(96.5 \mathrm{~cm}$.)). The heart was regular, rapid, with a tapping apical impulse, a loud apical first sound, and accentuation of the second sound. There was a rumbling mitral mid-diastolic, a presystolic, and a faint short, early systolic murmur. The electrocardiogram showed evidence of right ventricular hypertrophy and bifid $P$ waves in lead V3 (Fig. 1). The chest radiograph

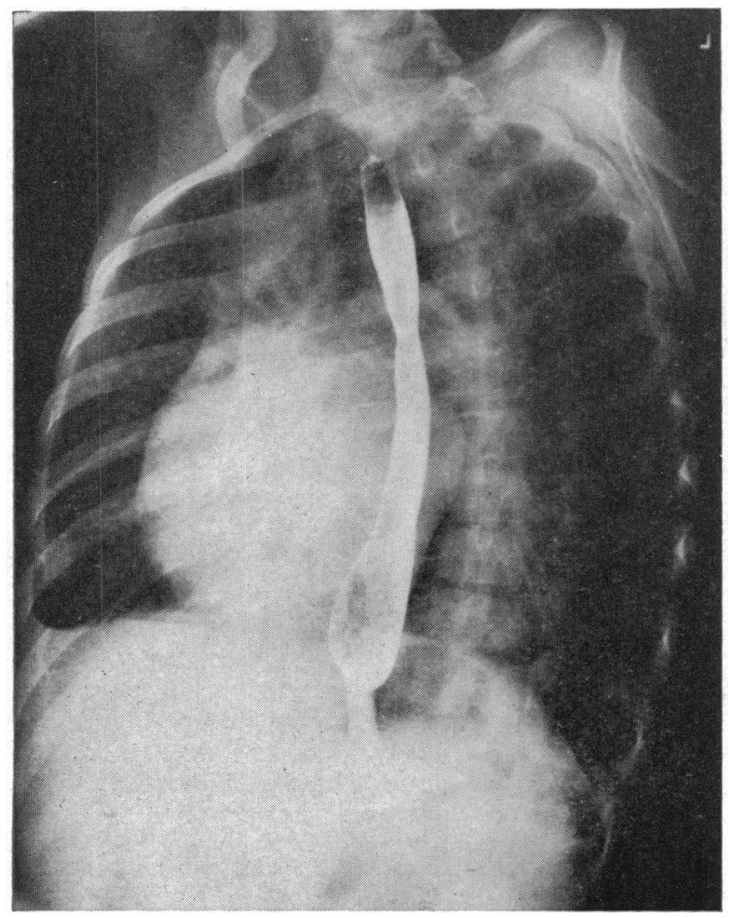

Fig. 2.-Left anterior oblique view of the chest showing prominence of the upper left atrial segment of the posterior border without prominence of the lower left ventricular segment (Case 4). revealed an enlarged heart with a prominent left atrium (Fig. 2). The lung vessels were congested and lymphatic lines were present. The pulmonary arterial pressure was $45-50 / 22 \mathrm{~mm}$. $\mathrm{Hg}$ and the wedge pressure $20 \mathrm{~mm}$. $\mathrm{Hg}$.

In 1964 a mitral valvotomy was performed under direct vision, using cardio-pulmonary bypass. Biopsy of the left atrium showed slight hypertrophy of the myocardial fibres and a little endocardial sclerosis. The child's immediate post-operative state was satisfactory, but he developed mitral regurgitation which became severe. This was successfully corrected at a second operation 12 weeks later. Evidence of pulmonary hypertension and associated breathlessness gradually diminished in the weeks following operation and progressed to complete freedom from symptoms, full exercise tolerance, and a heart of normal size with normal sounds and only a faint short systolic murmur at the apex.

Case 5. This girl was first seen at the age of 3 months because she had failed to thrive. She had a persistent ductus arteriosus which was closed when she was 4 months old. She was readmitted in 1963 at the age of nearly 5 years with congestive heart failure and suspected mitral stenosis. Response to digitalis and diuretics was satisfactory. She was small (weight $30 \mathrm{lb}$. (13.6 kg.), height $38 \mathrm{in}$. $(96.5 \mathrm{~cm}$.)), with a malar flush and a chest deformity consisting of a prominent sternum and indrawn lower ribs. The cardiac impulse was of right ventricular type. Tachycardia made it difficult to time the murmurs, but phonocardiography showed that, in addition to loud first mitral and second pulmonary sounds, there was a presystolic murmur at the apex and an early diastolic murmur at the left sternal edge. Electrocardiogram showed sinus rhythm, bifid $\mathbf{P}$ waves in V3, and evidence of right ventricular hypertrophy (Fig. 1). Radiography showed cardiomegaly, a prominent left atrium, dilated pulmonary artery, and pulmonary vascular congestion with lymphatic lines (Fig. 3). Mean left atrial pressure, measured in 1964 by transseptal puncture from the right atrium, was $15 \mathrm{~mm}$. $\mathrm{Hg}$, and the pulmonary arterial pressure was $92 / 50 \mathrm{~mm}$. $\mathrm{Hg}$. Cineangiocardiography showed a large, slowly emptying left atrium. The mitral valve bulged into the left ventricle during left ventricular filling and was flattened or concave during ventricular systole, confirming the diagnosis of mitral stenosis.

At operation in 1965 the valve was funnel-shaped with thick rubbery cusps. Its orifice measured $1.0 \mathrm{~cm}$. in diameter, and in the area where the postero-medial commissure should have been the cusps were fused to anomalous chordx tendinex. After splitting the valve under direct vision, the orifice measured $2.5 \mathrm{~cm}$. in diameter but left atrial pressure remained high $(14 \mathrm{~mm}$. $\mathrm{Hg}$ ). Right ventricular pressure fell only from 50 to $45 \mathrm{~mm}$. Hg. Left ventricular pressure was $100 \mathrm{~mm}$. $\mathrm{Hg}$. There was difficulty in maintaining an adequate systemic pressure at the end of perfusion. Acute pulmonary cedema developed and positive pressure ventilation was necessary. She died within a few hours of 


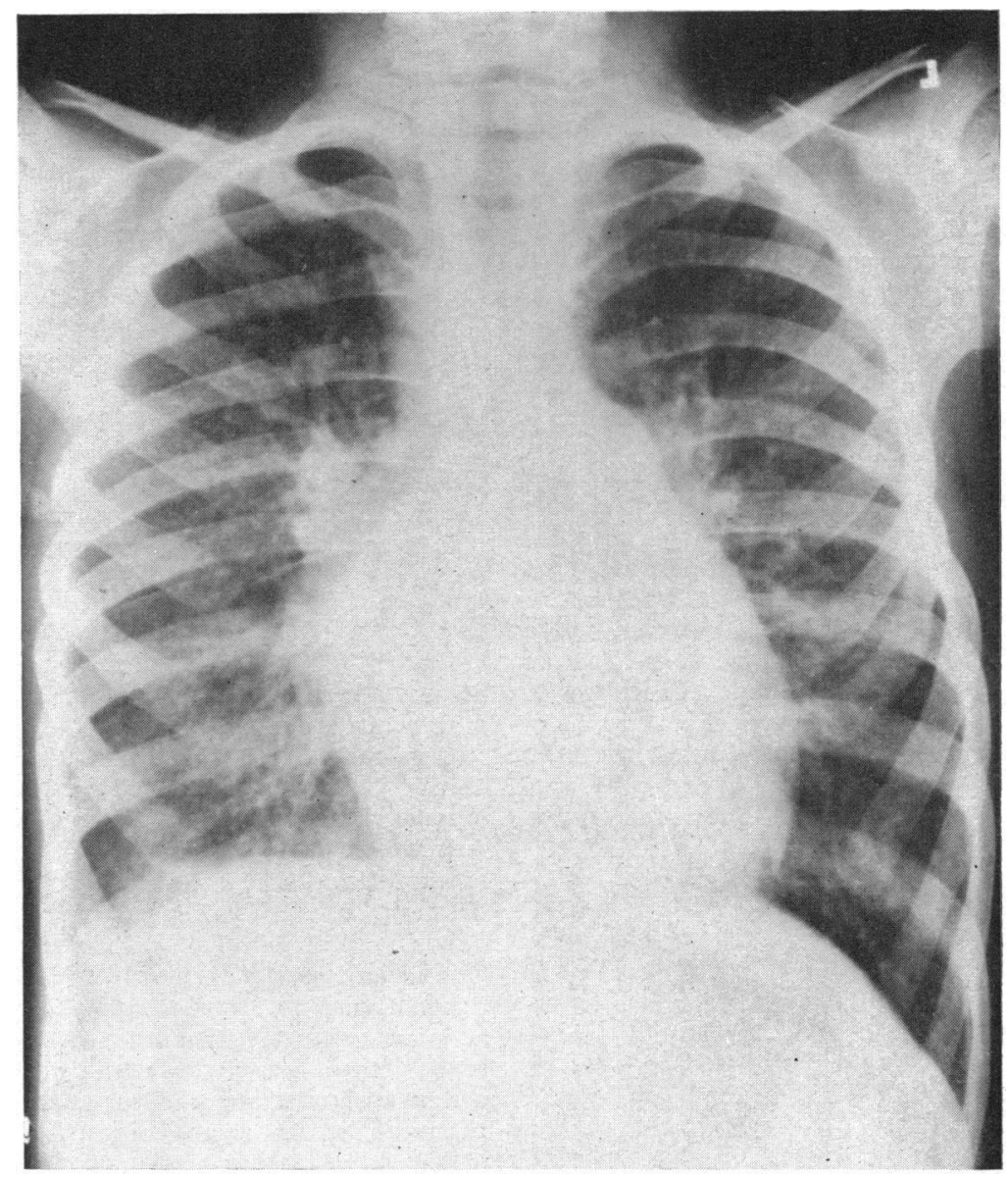

FIG. 3.-Postero-anterior view of chest showing a dilated pulmonary artery, prominence of the left middle segment below the pulmonary artery, and pulmonary vascular congestion (Case 5).

returning to the ward. At necropsy (Dr. H. C. Cameron) the mitral valve measured $6.2 \mathrm{~cm}$. in circumference (the pre-operative circumference was judged to be $3.7 \mathrm{~cm}$.). The valve consisted of one large irregular cusp which was attached to half the circumference of the valve ring, the remainder being "a nodular rim". One of the chordæ was split longitudinally. The aortic valve was normal. Both atria were dilated and both ventricles were hypertrophied. The main pulmonary artery was dilated and there were a few small foci of atheroma. Microscopy of the lungs showed great hypertrophy of the large and small muscular arteries with reduplication of the internal elastic lamina. The intima showed varying degrees of thickening and fibrosis, sufficient in some areas to cause complete occlusion of the lumen with disruption of the elastic laminæ. Although no active necrotizing arteritis was seen, the appearance was that of severe prolonged pulmonary hypertension. Near the apex of the left ventricle, anteriorly, was a small septal defect and a local patch of endocardial thickening. Generalized fibro-elastosis was absent.
Case 6. In 1964 at the age of 13 months this boy began to get frequent respiratory infections. When he was 20 months old he developed the first of a series of attacks of paroxysmal supraventricular tachycardia which precipitated congestive heart failure. He was small (weight $19 \mathrm{lb}$. $(8.6 \mathrm{~kg}$ ), height 29 in. $(73 \mathrm{~cm}$.)). The heart was enlarged. There was a loud mitral first sound and a murmur which could not be accurately timed on account of tachycardia. Apical presystolic and middiastolic murmurs were demonstrated by phonocardiography. X-ray examination confirmed cardiomegaly, a prominent left atrium, and pulmonary vascular congestion, and the cardiogram showed a peaked $P$ wave in V1 and right ventricular preponderance (Fig. 1). The pulmonary arterial pressure was $60 / 30 \mathrm{~mm}$. $\mathrm{Hg}$. Mitral stenosis was confirmed by cine-angiocardiography, the right ventricle being enlarged and the large left atrium slow to empty. He has responded well to medical treatment, digoxin controlling the paroxysmal tachycardia. Operation will be considered when he is about 5 years of age. 
Case 7. A girl of 6 was found to have an abnormal heart in 1965 when she had bronchopneumonia. She had had no previous symptoms and had developed normally (weight $37 \mathrm{lb}$. (16.8 kg.), height $44 \frac{1}{2}$ in. (112 cm.)). Slight cardiac enlargement was present with a tapping apical impulse and a mid-diastolic thrill. An accentuated mitral first sound was associated with a presystolic and a rough mid-diastolic murmur. The only electrocardiographic abnormality consisted of bifid $\mathbf{P}$ waves, particularly obvious in V2 and V3 (Fig. 1). There was radiological evidence of right ventricular and left atrial enlargement, though the osophagus was not displaced. Pulmonary vascular congestion was marked and lymphatic lines were present. Pulmonary arterial pressure was $65-70 / 30 \mathrm{~mm}$. $\mathrm{Hg}$ and the wedge pressure was $25 \mathrm{~mm}$. Hg. Selective cine-angiocardiography showed a large, slowly emptying left atrium, a "halo" in the area of the mitral valve, and a rather small left ventricle. The inferior vena cava ran an anomalous course, giving support to the view that the condition was of congenital origin.

Response to medical treatment has been only fair but the parents have been unwilling to allow the child to return to hospital.

Case 8. A male infant was admitted to hospital in 1965 at the age of 2 months. He weighed $7 \mathrm{lb} .5 \mathrm{oz}$. (3317 g.) at birth and proved difficult to feed, vomiting frequently, and had only just regained his birthweight. Cyanosis was observed soon after birth but there was no difference in colour of hands and feet. Respiration was difficult and there was recession of the lower part of the chest. Venous engorgement and hepatic enlargement were noted. The heart was enlarged and the apical impulse was left ventricular in type. There was triple rhythm, and not until the heart failure had been controlled by digoxin and diuretics was it possible to hear a soft short mid-systolic murmur. Bifid $\mathbf{P}$ waves were seen in leads I, II, V3, and V5 of the cardiogram (Fig. 1); the ventricular complexes were normal. Radiographs showed cardiomegaly with pulmonary vascular and lymphatic congestion, and venous angiocardiography demonstrated that blood flowed from the right ventricle into the pulmonary artery and directly through a ductus arteriosus into the descending aorta. Blood passing to the lungs returned to a large left atrium which emptied slowly. The left ventricle indented the right ventricle and the aorta appeared to be normal in size. As response to medical treatment was inadequate, the mitral valve was dilated with artery forceps. Biopsy of the left atrium showed moderate fibro-elastic endocardial sclerosis. A systolic thrill, felt at operation, suggested that the aortic valve was also stenotic.

The baby's colour improved considerably after operation and the weight increased slowly during the next month to a maximum of $8 \mathrm{lb} .2 \mathrm{oz}$. (3685 g.). Thereafter there was a gradual deterioration with a fall of weight to $7 \mathrm{lb} .6 \mathrm{oz}$. (3345 g.) ten weeks after operation. As respiratory difficulties were considerable and signs of aortic stenosis were clearly evident, cardiac catheterization was undertaken. Aortic pressure was $65 / 53 \mathrm{~mm}$. $\mathrm{Hg}$ (catheter introduced through the brachial artery).
The left ventricle could not be entered from above but contrast medium was injected and gave the typical dome and jet appearance of congenital aortic stenosis. A catheter introduced into a vein at the groin entered the pulmonary artery $(55 / 35 \mathrm{~mm}$. $\mathrm{Hg}$ ) and passed through the ductus into the descending aorta $(45 / 30 \mathrm{~mm} . \mathrm{Hg})$. The catheter was also passed through the foramen ovale into the left ventricle $(140-155 / 7 \mathrm{~mm}$. $\mathrm{Hg})$.

In view of the severity of the stenosis the aortic valve was dilated using the transventricular approach. During the operation the aortic pressure rose from 50/40 to $115 / 75 \mathrm{~mm}$. $\mathrm{Hg}$, and six weeks later the arm blood pressure was $80 / 50 \mathrm{~mm}$. $\mathrm{Hg}$. A systolic ejection murmur persisted. Unfortunately a wound infection led to generalized septicæmia and the child died 7 weeks after the final operation. At necropsy endocardial fibroelastosis of the left atrium was confirmed, but there was none of the left ventricle. The mitral valve showed residual narrowing but the aortic stenosis had been completely relieved; both valves were affected by active bacterial endocarditis. The ductus arteriosus was still open.

\section{DisCUSSION}

Rheumatic carditis is very rare in early childhood and mitral stenosis at this age is likely to be of congenital origin (Bland, White, and Jones, 1935). Mannheimer, Bengtsson, and Winberg (1952) attributed the defect to primary endocardial fibrosis. On the other hand, Ferencz, Johnson, and Wiglesworth (1954), who found that the endocardial changes were confined to the left atrium in 7 out of 8 cases, concluded that they were likely to be the result of an obstructive lesion rather than its cause. In our series of 8 cases fibro-elastosis of the left atrial endocardium was found in 4 out of 6 cases examined; none had evidence of rheumatic involvement. In the 3 cases which came to necropsy fibro-elastosis of both left atrium and left ventricle was found in one, of the left atrium alone in one, and of neither chamber in the third; in the latter case a small area of endocardial thickening in the left ventricle was attributed to a "jet effect".

Breathlessness, recurrent bronchitis, and failure to grow properly were early symptoms in 7 patients. This tendency for symptoms to be noticed in infancy was mentioned by Baker et al. (1962) and by Daoud et al. (1963). A desire to sit up in bed was a distinctive feature sufficiently uncommon in other forms of congenital heart disease to suggest the diagnosis and to distinguish mitral stenosis from other conditions with an apical mid-diastolic murmur.

Hæmoptysis is not uncommon in rheumatic mitral stenosis. Its absence in congenital mitral stenosis may perhaps be due to high pulmonary vascular resistance which protects the pulmonary capillaries (Thompson and Stewart, 1951). 
Physical underdevelopment was striking. Four children had an abnormally-shaped chest with an increased antero-posterior diameter and a deformity reminiscent of Harrison's sulcus. We attribute this to traction by the diaphragm on the lower ribs due to low pulmonary compliance associated with pulmonary venous hypertension and obstructive airway disease resulting from attacks of bronchitis.

Sinus rhythm was the rule in early childhood, but a girl of 13 developed atrial fibrillation during the terminal phase of her illness. Patients described by Nadas (1963), Baker et al. (1962), and Daoud et al. (1963) were all in sinus rhythm. Right ventricular hypertrophy was characterized in older children by a parasternal systolic heave and a tapping apex beat, but these signs were difficult to elicit in infants. A loud first sound and accentuated pulmonary valve closure were notable features. An opening snap was not heard though it has been noted in other reports (Baker et al., 1962). Nadas (1963) attributed the absence of an opening snap to the rubbery nature of the mitral valve.

Ferencz et al. (1954) heard a diastolic murmur in only 1 of their 9 cases and did not find auscultation helpful. By contrast we heard an apical middiastolic and presystolic murmur in 7 of our 8 patients and did not suspect the diagnosis when the characteristic murmur was lacking (Case 8). Both Hauck et al. (1959) and Kjellberg et al. (1959) had the same experience.

Tachycardia and the murmurs of pulmonary incompetence sometimes made it difficult to time the characteristic apical murmurs. For example, when the heart rate is rapid, a mid-diastolic murmur may merge into a presystolic murmur and give the mistaken impression of a long systolic murmur. Phonocardiography has provided useful information in cases of doubt.

Electrocardiograms were of value in diagnosis when there were broad bifid $P$ waves, seen most commonly in V3. Right ventricular preponderance was observed in 6 of the 8 children (Fig. 1).

Radiological examination showed a heart size that varied from normal to considerable enlargement. The pulmonary artery segment was often prominent and, after infancy, 6 children had a prominence in the left middle segment a little suggestive of left atrial enlargement; the left bronchus was raised in only 2 children. However, in the left anterior oblique view there was a characteristic appearance in 5: this was produced by prominence of the left atrial segment, i.e. the upper part of the posterior border, without prominence of the left ventricular segment, i.e. the lower part of the posterior border (Fig. 2). In the lateral view, the area of cardiac contact with the diaphragm was sometimes notably small, another manifestation of the relatively small left ventricle.

While under observation, all 8 children at some time showed congestive changes in the lungs. There was often hazy pulmonary opacity associated with traces of fluid in the interlobar fissures and against the chest wall. Four children showed basal lines of lymphatic congestion and large pulmonary veins were sometimes detectable.

Radiological signs of left heart failure are relatively uncommon in congenital heart disease and their presence should always bring congenital mitral stenosis under consideration. When associated with left atrial enlargement without a large left ventricle this becomes very probable.

Angiocardiography with venous or right heart injections was carried out in all the children. This showed a rather slow passage through the left heart and distinct stasis in the large left atrium. Compared with the large atrium the left ventricle and aorta seemed small in 7 of the 8 children, and the one child with a large left ventricle had complicating aortic stenosis. In cases of mitral regurgitation which we have investigated there has been a large left atrium and a large left ventricle and a to-and-fro movement of contrast between the two chambers. In two patients the first contrast medium to enter the left atrium from the pulmonary veins "floated" into the non-opacified blood already filling it. This was best seen in the right anterior oblique projection. In three patients this view also showed signs directly suggesting mitral stenosis, but the best way to demonstrate these direct signs is to inject contrast medium into the left atrium. In one patient injections were made into the left ventricle (entered via the right brachial artery and aorta) to exclude mitral regurgitation, and in this child the left atrium was also injected after transseptal puncture. In the right anterior oblique projection, before the mitral valve opened it appeared as a flat area. As opening began a tapering cone of contrast medium entered the left ventricle and in subsequent cycles this cone of greater density was seen several times within the contrast medium in the ventricle, clternating with a flat area of the left atrium when the valve closed. Injections with and without $15^{\circ}$ of tilt of the central rays towards the head were equally effective in demonstrating the stenosis. This limitation of the entering medium to a cone was not seen in children with predominant mitral regurgitation when they were examined in the same way.

Reports of surgical treatment in infants with mitral stenosis have not been encouraging (Starkey, 1959; Daoud et al., 1963). In congenital mitral 
stenosis the annulus is not usually reduced in size but the valve is funnel-shaped with thick leathery cusps held down by short thick fused chordæ tendinex. Commissures are absent or rudimentary. These features make digital valvotomy difficult. It was used in one of our patients (Case 1) in 1952 (Bower et al., 1953). This child survived and improved for several years but a second operation was required in 1959, using cardiopulmonary bypass and direct vision. Instrumental dilatation through the left atrium was used in Case 2 and appeared to be satisfactory, though the child died suddenly 36 hours after operation. Instrumental dilatation first of the mitral and later of the aortic valve was used successfully in another infant (Case 8). Regurgitation did not occur as a result of these operations.

Open mitral valvotomy was undertaken in three of our older patients. The results were satisfactory in two, though one of these developed gross mitral regurgitation which needed correction at a second operation. The third child died after a technically difficult operation.

Open operation seems preferable if the child is in the older age-group because direct vision should provide a better chance of obtaining a good surgical result, but when Ferencz et al. (1954) reviewed 43 reported cases they found that only one had lived beyond the age of 3 years. This high mortality in early life may have been due to associated lesions such as fibro-elastosis, aortic stenosis, aortic coarctation, persistent ductus arteriosus, and septal defects, or to the respiratory infections commonly associated with heart failure. The introduction of antibiotics may explain why, in 1963, Daoud et al. were able to report 7 patients who had survived early childhood, and why we should have been able to follow 6 children into the older age-group.

Our experience suggests that congenital mitral stenosis causing heart failure in infancy is unlikely to respond to medical treatment, whereas heart failure occurring later in childhood usually responds to digitalis and diuretics unless atrial fibrillation has developed.

We are not sure how quickly pulmonary vascular changes progress. A slight increase only of pulmonary arterial pressure occurred in three children, each examined twice by cardiac catheterization at intervals of $1 \frac{1}{2}, 7$, and $7 \frac{1}{2}$ years, respectively. However, we do not know what changes may have developed in the pulmonary blood vessels and the lung parenchyma during these periods, because we did not obtain serial records of pulmonary vascular resistance or serial lung biopsies. Harris and Heath (1962) described changes which they attributed to raised pulmonary venous pressure and inferred that severe pulmonary arterial damage, with fibrosis of the intima and hypertrophy of the media, occurred only in the late stages of mitral stenosis. But Ferencz and her colleagues (1954) commented on the early development of vascular changes, and we have seen severe and apparently irreversible damage in the lung vessels of a child aged 7 years. We, therefore, conclude that, when a child with congenital mitral stenosis develops heart failure, operation should be advised unless medical treatment is rapidly effective.

\section{SUMMARY}

Eight patients with congenital mitral stenosis have been reviewed. Orthopncea, frequent respiratory infections, and failure to thrive were the usual symptoms. Phonocardiography proved a valuable aid, especially in infants, when tachycardia and associated pulmonary incompetence made timing of the murmurs difficult. The electrocardiogram was helpful when there was evidence of right ventricular hypertrophy or bifid $\mathrm{P}$ waves were present. Radiological examination frequently showed a characteristic picture of congestive changes in the lungs associated with a large left atrium but a relatively small left ventricle. Angiocardiography, as well as excluding other lesions, confirmed the relative sizes of the left heart chambers and showed stasis in the large left atrium. Left-sided contrast injection, and the right oblique projection, gave optimal conditions for direct demonstration of the stenosed mitral valve.

Associated abnormalities were endocardial fibroelastosis (demonstrated in 4 of 6 cases examined), persistent ductus arteriosus in 3 cases, with reversed shunt in 2 , aortic stenosis in 1 case, ventricular septal defect in another, and an abnormal inferior vena cava in 1 case.

In 3 cases, each catheterized on two occasions, there was no evidence that pulmonary hypertension increased significantly during childhood, but pulmonary vascular changes may be severe. When cardiac failure occurs it is inadvisable to delay surgery, despite the risks, except in cases where pulmonary arterial pressure is only slightly raised and in patients who respond rapidly to medical treatment.

\section{REFERENCES}

Baker, C. G., Benson, P. F., Joseph, M. C., and Ross, D. N. (1962). Congenital mitral stenosis. Brit. Heart f., 24, 498.

Bland, E. F., White, P. D., and Jones, T. D. (1935). The development of mitral stenosis in young people. Amer. Heart F., 10, 995. 
Bower, B. D., Gerrard, J. W., d'Abreu, A. L., and Parsons, C. G. (1953). Two cases of congenital mitral stenosis treated by valvotomy. Arch. Dis. Childh., 28, 91 .

Daoud, G., Kaplan, S., Perrin, E. V., Dorst, J. P., and Edwards, F. K. (1963). Congenital mitral stenosis. Circulation, 27, 185.

Ferencz, C., Johnson, A. L., and Wiglesworth, F. W. (1954). Congenital mitral stenosis. Circulation, 9, 161.

Harris, P., and Heath, D. (1962). The Human Pulmonary Circulation, p. 215. Livingstone, Edinburgh.

Hauck, A. J., Rudolph, A. M., Starkey, G. W. B., and Nadas, A. S. (1959). Proc. New Engl. cardiovas. Soc., 1958-59, 27, 18.
Kjellberg, S. R., Mannheimer, E., Rudhe, U., and Jonsson, B. (1959). Diagnosis of Congenital Heart Disease, 2nd ed., p. 752. Year Book Publishers, Chicago.

Mannheimer, R., Bengtsson, E., and Winberg, J. (1952). Pure congenital mitral stenosis due to fibro-elastosis. Cardiologia (Basel), 21, 574.

Nadas, A. S. (1963). Pediatric Cardiology, 2nd ed., p. 587. Saunders, Philadelphia and London.

Starkey, G. W. B. (1959). Surgical experiences in the treatment of congenital mitral stenosis and mitral insufficiency. F. thorac. cardiovasc. Surg., 38, 336.

Thompson, A. C., and Stewart, W. C. (1951). Hemoptysis in mitral stenosis. F. Amer. med. Ass., 147, 21. 